\title{
Dynamics of Cryogenic Jets: Non-Rayleigh Breakup and Onset of Nonaxisymmetric Motions
}

\author{
A. V. Boukharov, ${ }^{1}$ M. Büscher ${ }^{2, *}$ A. S. Gerasimov, ${ }^{3}$ V. D. Chernetsky, ${ }^{3}$ P. V. Fedorets, ${ }^{2,3}$ I. N. Maryshev, ${ }^{1}$ \\ A. A. Semenov, ${ }^{1}$ and A. F. Ginevskii ${ }^{1}$ \\ ${ }^{1}$ Moscow Power Engineering Institute, Ul. Krasno-Kazarmennaja 13, 117218 Moscow, Russia \\ ${ }^{2}$ Institut für Kernphysik, Forschungszentrum Jülich, 52425 Jülich, Germany \\ ${ }^{3}$ Institute for Theoretical and Experimental Physics, B. Cheremushkinskaya 25, 117218 Moscow, Russia
}

(Received 1 June 2007; published 2 May 2008)

\begin{abstract}
We report development of generators for periodic, satellite-free fluxes of monodisperse drops with diameters down to $10 \mu \mathrm{m}$ from cryogenic liquids such as $\mathrm{H}_{2}, \mathrm{~N}_{2}, \mathrm{Ar}$, and $\mathrm{Xe}$ (and, as a reference fluid, water). While the break up of water jets can be described well by Rayleigh's linear theory, we find jet regimes for $\mathrm{H}_{2}$ and $\mathrm{N}_{2}$ which reveal deviations from this behavior. Thus, Rayleigh's theory is inappropriate for thin jets that exchange energy and/or mass with the surrounding medium. Moreover, at high evaporation rates, the axial symmetry of the dynamics is lost. When the drops pass into vacuum, frozen pellets form due to surface evaporation. The narrow width of the pellet flux paves the way towards various industrial and scientific applications.
\end{abstract}

DOI: 10.1103/PhysRevLett.100.174505

PACS numbers: 47.15.Uv, 07.20.Mc, 29.25.Pj, 52.50.Dg

Drop formation from incompressible liquids is ubiquitous in daily life and technology, and such processes have been a focus of scientific investigations for almost two centuries [1-6]. Even the simple case of jets emanating from a vibrating nozzle leads to a broad spectrum of drop sizes, and the existence of satellite drops [7]. However, for technologies like ink-jet printing [8], laser-plasma uv sources [9], compact laser-based particle accelerators [10], accelerator experiments [11], or space operations [12], the drop sizes and rates must be highly homogeneous.

The disintegration of a slow cylindrical jet is caused by the growth of perturbations initiated at the ejection nozzle. An axially symmetric perturbation leads to jet breakup when its amplitude becomes equal to the jet radius. This domain is commonly denoted as the Rayleigh regime. In 1878 Lord Rayleigh described fluids in the limit of zero viscosity [2], while his 1892 paper [3] concerns the opposite limit where inertial effects are negligible compared to viscous ones. Rayleigh's work was extended by Chandrasekhar [4] to arbitrary viscosities. It has been shown [7] that the measurement of breakup lengths, e.g., for the forced break up of water jets with precise control of the nozzle-perturbation frequency, allows for precise tests of linear theories, although the final stage of capillary pinching is nonlinear [13].

Experimentally, the selection of a well-defined initial surface-perturbation amplitude and a certain frequency is commonly achieved by using piezoelectric transducers. Most of the existing data have been obtained for water (or water-based ink) and fluids of higher viscosities, with jets emanating into air at normal pressure.

In this Letter we report on first quantitative measurements of the break up of cryogenic and water jets, injected into the same gaseous substance close to triple-point (TP) conditions $\left(63 \mathrm{~K}\right.$ and 125 mbar for $\mathrm{N}_{2}, 14 \mathrm{~K}$ and 70 mbar for $\mathrm{H}_{2}$ ). In doing so we can widen the experimentally accessible parameter space, while keeping the Reynolds number $\operatorname{Re}=\left(\rho v_{\text {jet }} R_{0}\right) / \mu$ (which represents the relative importance of inertial and viscous forces) and the Ohnesorge number $\mathrm{Oh}=\mu /\left(\sigma \rho R_{0}\right)^{1 / 2}$ (viscous forces and surface tension) in the same range as for the existing water data. $\rho(\mu, \sigma)$ denote the fluid density (viscosity, surface tension), and $v_{\text {jet }}\left(R_{0}\right)$ the jet velocity (initial jet radius). At TP pressures and jet velocities of a few $\mathrm{m} / \mathrm{s}$, aerodynamic interactions with the ambient medium are small and, thus, we are close to the ideal case of an inviscid jet breaking up in vacuum and our data allow for clean tests of Rayleigh's theory.

According to the linear theory, a small surface perturbation $\delta$ (measured in units of $R_{0}$ ) of a jet moving in the $z$ direction, imposed at the nozzle exit $(z=0)$ with frequency $f$, grows exponentially towards the jet axis $[2,14]$. Then, the minimum (over time) necking of the jet $R(z)$ at a certain distance $z$ from the nozzle is

$$
\frac{R(z)}{R_{0}}=1-\delta \exp \left(\frac{\gamma / \gamma_{0}}{\mathrm{Oh} \operatorname{Re}} \frac{z}{R_{0}}\right)
$$

The dimensionless growth rate $\gamma / \gamma_{0}$ depends on the wavelength $\lambda$ of the perturbation and can be expressed in terms of a reduced wave number $X=2 \pi R_{0} / \lambda=2 \pi R_{0} f / v_{\text {jet }}$ for $X<1$ and $\operatorname{Re} \mathrm{Oh} \gg 1$ :

$$
\frac{\gamma}{\gamma_{0}}=-\frac{3}{2} \mathrm{Oh} X^{2}+\sqrt{\frac{9}{4} \mathrm{Oh}^{2} X^{4}+\frac{1}{2} X^{2}\left(1-X^{2}\right)},
$$

with $\gamma_{0}=\operatorname{Oh}\left(\sigma / \mu R_{0}\right)$. Figure 1 shows a plot of Eq. (2) for water, $\mathrm{N}_{2}$, and $\mathrm{H}_{2}$ jets; linear theory predicts a practically identical $X$ dependence of $\gamma / \gamma_{0}$ for all three liquids. If $\mathrm{Re} \mathrm{Oh}$ approaches unity, the theory tends to underestimate $\gamma / \gamma_{0}$; see, e.g., the discussion by Kalaaji et al. [7].

Since a jet breaks into drops for $R(z)=0$, the jet length $L_{\text {jet }}$ results from Eq. (1) as 


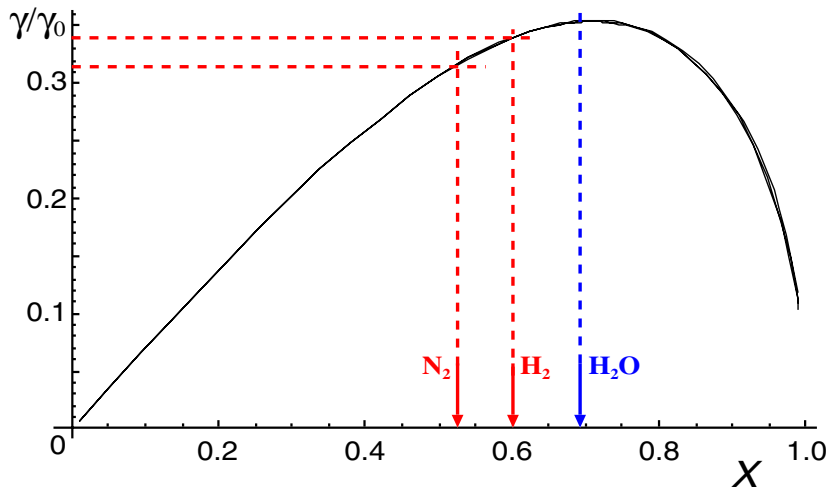

FIG. 1 (color online). Dimensionless growth rate $\gamma / \gamma_{0}$ as a function of the reduced wave number $X$ in linear theory. For $\mathrm{N}_{2}$ and $\mathrm{H}_{2}$ two (practically identical) curves have been calculated with Eq. (2) using the Oh values from Table I. For water a typical Ohnesorge number $\mathrm{Oh}=0.02$ has been chosen, since $\gamma / \gamma_{0}$ behaves similarly at $\mathrm{Oh}<0.1$. The arrows indicate the $X$ values for which we have observed satellite-free drop production.

$$
L_{\text {jet }}=\frac{R_{0}}{\gamma / \gamma_{0}} \operatorname{Re} \operatorname{Oh} \ln (1 / \delta)=\frac{v_{\text {jet }}}{\gamma / \gamma_{0}} \sqrt{\frac{\rho R_{0}^{3}}{\sigma}} \ln (1 / \delta) .
$$

Relations (1)-(3) have been experimentally verified in numerous studies with water or viscous fluids $[7,15]$. Satellite-free and monodisperse water-drop production has also been reported: for not too large values of $\delta$ $(\lesssim 0.01)$ it only occurs at maximum values of $\gamma / \gamma_{0}$, i.e., at $X_{\max }=0.69[16,17]$.

Quantitative studies of forced jet breakup depend crucially on the suppression of unwanted nozzle vibrations which can, e.g., be caused by cold head units [18]. Thus our drop generator [19] is surrounded by a cryostat with baths of liquid $\mathrm{N}_{2}$ and He; see Fig. 2. Liquefaction of $\mathrm{H}_{2}$ is achieved in three stages: first, cooling with liquid $\mathrm{N}_{2}$, further cooling in a heat exchanger by evaporated $\mathrm{He}$, and final cooling in the condenser by cold $\mathrm{He}$ gas. For production of, e.g., $\mathrm{N}_{2}$ and Ar jets, cooling with liquid $\mathrm{N}_{2}$ is sufficient, while for water we use a dedicated generator without cooling cryostat. The temperatures along the main gas and liquid flows are controlled with an accuracy of $0.1 \mathrm{~K}$. From the condenser, the liquid passes through the vibrating nozzle and reaches the triple-point chamber (TPC) where the jet disintegrates into drops. The liquid temperature in the nozzle is maintained slightly below the boiling point and, according to simulation calculations, the temperature decrease in the jet until breakup from heat exchange in the TPC is below $0.2 \mathrm{~K}$ (cf. Table I). Therefore, the generation of semisolid domains can be excluded. The temperatures and pressures in the TPC are kept over several hours within $\pm 0.2 \mathrm{~K}( \pm 0.2 \mathrm{mbar})$ at the nominal values.

Two nozzle types have been utilized: glass nozzles in brass housings and such made from stainless steel. The former, with inner diameters of $12-30 \mu \mathrm{m}$ at the nozzle

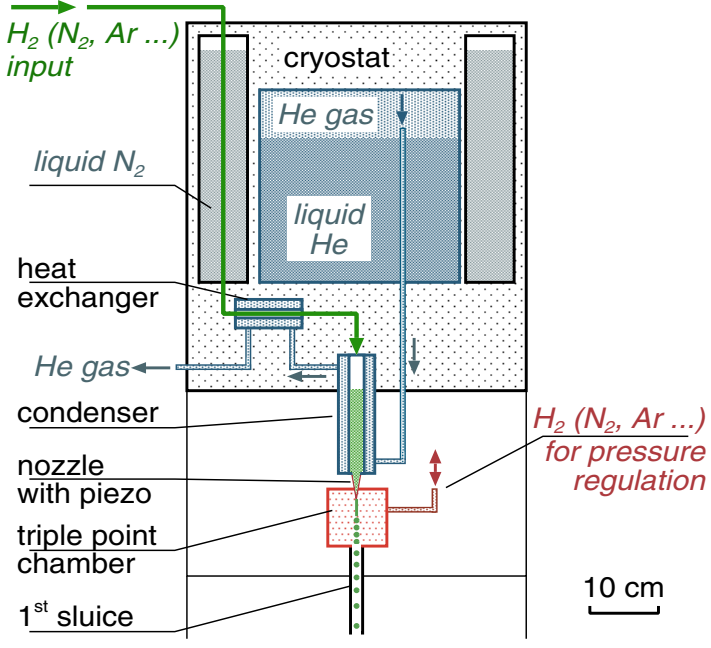

FIG. 2 (color online). Sketch of the drop generator. The cryostat (total height $\sim 0.8 \mathrm{~m}$, not drawn to scale) contains the cooling liquids $\left(\mathrm{N}_{2}\right.$ and $\left.\mathrm{He}\right)$ as well as the heat exchanger and the upper part of the condenser. These are cooled by a stream of evaporated $\mathrm{He}$ gas. The condenser houses a few $\mathrm{cm}^{3}$ of the cryogenic liquid which is driven through a nozzle into the TPC by overpressure. Constant pressure in the TPC is maintained by an auxiliary gas feeding.

tip, have the advantage of a smooth internal surface and allow one to look inside the channel during operation. The 16-30 $\mu \mathrm{m}$ steel nozzles offer high shape reproducibility and smaller length-to-diameter ratios of the holes, allowing operation with lower jet-driving pressures. The results presented here have been obtained with glass nozzles and pressures of $\sim 0.4-0.9$ bar for $\mathrm{H}_{2}$ and $\sim 1.0-1.5$ bar for $\mathrm{N}_{2}$. Higher pressures lead to higher jet velocities. The piezo-

TABLE I. Parameters of the jets from Fig. 3. $\gamma / \gamma_{0}$ is calculated with Eq. (2), the jet length $L_{\text {jet }}^{\text {Rayleigh }}$ with Eq. (3).

\begin{tabular}{lcc}
\hline \hline & $\mathrm{N}_{2}$ & $\mathrm{H}_{2}$ \\
\hline Density $\rho\left(\mathrm{kg} / \mathrm{m}^{3}\right)[20,21]$ & 823.7 & 64.04 \\
Static surface tension $\sigma(\mathrm{N} / \mathrm{m})[20,21]$ & 0.0094 & 0.0025 \\
Viscosity $\mu(\mathrm{mPas})[20,21]$ & 0.2113 & 0.0183 \\
TPC pressure $(\mathrm{mbar})$ & 300 & 130 \\
TPC temperature $(\mathrm{K})$ & 74 & 17 \\
Jet temperature $(\mathrm{K})$ & $77.2-77.0$ & $20.0-19.8$ \\
Nozzle frequency $f(\mathrm{kHz})$ & 26 & 38 \\
Jet diameter $2 R_{0}(\mu \mathrm{m})$ & 17 & 12 \\
Jet velocity $v_{\text {jet }}(\mathrm{m} / \mathrm{s})$ & 2.6 & 2.4 \\
Reduced wave number $X$ & 0.53 & 0.60 \\
Reynolds number $(\mathrm{Re})$ & 84 & 50 \\
Ohnesorge number $(\mathrm{Oh})$ & 0.026 & 0.019 \\
Re Oh & 2.2 & 1.0 \\
Growth rate $\gamma / \gamma_{0}$ & 0.31 & 0.34 \\
Initial perturbation $\delta$ & 0.02 & 0.02 \\
Expected jet length $L_{\text {jet }}^{\text {Rayleigh }}(\mu \mathrm{m})$ & 240 & 70 \\
Measured jet length $L_{\text {jet }}(\mu \mathrm{m})$ & 310 & 290 \\
\hline \hline
\end{tabular}


electric transducer allows us to excite sinusoidal nozzle vibrations in the range $f=1-60 \mathrm{kHz}$.

The jet decay has been observed with two perpendicularly placed CCD cameras and strobe lamps that are triggered synchronously to the nozzle, with a 10 times lower frequency, and a flash duration of $\sim 1.5 \mu \mathrm{s}$. The jet diameter $2 R_{0}$ has been assumed to be equal to the nozzle diameter which is correct to better than $\pm 10 \%$ [7]. This is confirmed by our measurement of jet diameters with the CCD cameras. $v_{\text {jet }}$ has been determined by measuring the wave-propagation velocity along the jet surface. The initial amplitude $\delta$ has been derived from reference measurements of water jet lengths using Eq. (3), as well as from direct amplitude measurements with an Michelson interferometer. See Ref. [15] for further details of the jet diagnostics.

Figure 3 shows the break up of $\mathrm{N}_{2}$ and $\mathrm{H}_{2}$ jets; for certain choices of the jet and TPC parameters (see Table I), satellite-free and monodisperse drop production is achieved. Astonishingly, we find it at $X=0.53$ and $X=$ 0.60 , respectively, i.e., for different wave numbers as compared to water. Since this is off the maximum from Fig. 1, it suggests an $X$ dependence of $\gamma / \gamma_{0}$ different from Eq. (2). In order to further test a possible deviation from Rayleighlike behavior, we have checked the validity of Eqs. (1)-(3) in more detail.

The last two lines of Table I compare the measured and predicted jet-decay lengths; it is seen that the cryogenic jets are much longer than expected. This cannot be explained by a variation of the parameters that enter Eq. (3). For example, an unphysical value of $\delta=10^{-8}$ would be required to yield the large decay length of the $\mathrm{H}_{2}$ jet. The dynamic surface tension can differ from the static values for $\sigma$ quoted in Table I; it had to be, however, 20 (2) times smaller to explain the observed $\mathrm{H}_{2}\left(\mathrm{~N}_{2}\right)$ jet lengths which seems unreasonable.

An explanation of the obvious violation of Rayleigh's theory might be disturbances like temperature, pressure,
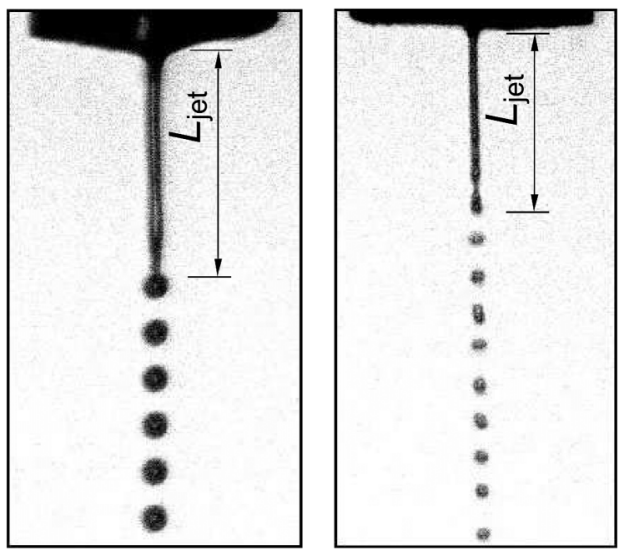

FIG. 3. First observation of satellite-free and monodisperse disintegration of $\mathrm{N}_{2}$ (left) and $\mathrm{H}_{2}$ (right) jets. In the upper edge of the photos the tip of the vibrating nozzle can be seen. and surface-tension variations as well as liquid-gas transitions. It has, e.g., been predicted [22] that evaporation and condensation decrease $L_{\text {jet }}$ (which seems to contradict the large measured $L_{\text {jet }}$ values from Table I) and, for large evaporation rates, satellite production can be suppressed. One may presume that for our jets evaporation is strong since in the TPC jets and ambient gas coexist close to the vapor-pressure curve. In order to test the effect of evaporation we have enhanced it by reducing the jet velocities or the TPC pressure (keeping the other parameters constant). This leads to a phenomenon, to our knowledge not reported until now. The jets slowly move from their vertical flux direction to one off the nozzle symmetry axis while preserving their smooth surfaces. The direction they choose seems to be random. However, once a jet has arrived at a certain stable configuration it may stay there for seconds; only at very small jet-bending radii are frequent abrupt changes of the jet directions observed. As an example we show in Fig. $4 \mathrm{~N}_{2}, \mathrm{H}_{2}$, and water jets. Even backbending is observed in some cases. Systematic studies reveal larger jet deflections from the vertical axis with decreasing jet diameter and velocity or TPC pressure. We note that asymmetric heating has been employed by another group to deflect liquid microjets [23]; however, we report spontaneous deflection of symmetrically produced jets.

When the drops leave the TPC through a 1st sluice into a subsequent chamber $\left[p=O\left(10^{-2}\right)\right.$ mbar], they freeze to pellets due to strong surface evaporation, and are accelerated by the gas flow from the TPC. Then the pellets pass a 2nd sluice and a 2nd chamber $\left[O\left(10^{-4}\right)\right.$ mbar], and finally reach a dummy scattering chamber through a thin tube [ $\sim 2 \mathrm{~cm}$ ], which is located $1.2 \mathrm{~m}$ downstream of the TPC. In order to minimize turbulences of the gas flow in the 1st sluice (and, thus, distortions of the produced pellets), it has a circular cross section with a radius that decreases exponentially to $600 \mu \mathrm{m}$ in flight direction. For pellet observation two CCD cameras have been positioned at the outlet of the 1st sluice or at the dummy chamber. Stable $\mathrm{H}_{2}$ and $\mathrm{N}_{2}$ pellet production with diameters $20-40 \mu \mathrm{m}$ (the pellet diameter is about two nozzle diameters) has been observed. Deviations from the mean pellet diameter are below $1 \%(10 \%)$ over periods of few seconds (hours). The average velocity of $30 \mu \mathrm{m}$ pellets
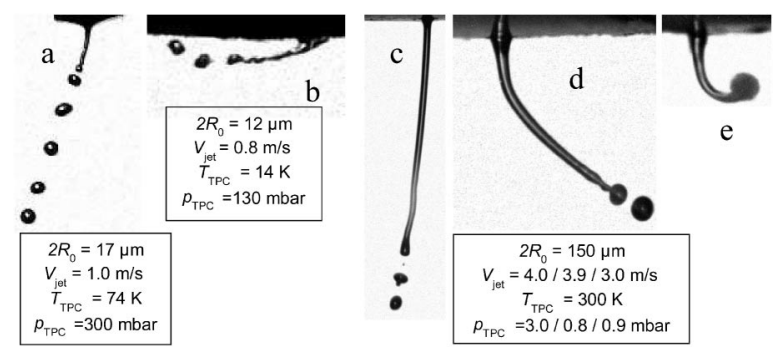

FIG. 4. First observation of nonaxisymmetric $\mathrm{N}_{2}$ (a), $\mathrm{H}_{2}$ (b), and water (c)-(e) jets. 
amounts to $\sim 70 \mathrm{~m} / \mathrm{s}$. The radial displacement of the pellets from their nominal flight path in the dummy chamber of about $\pm 200 \mu \mathrm{m}$ has been extrapolated from the angular pellet distributions measured behind the 1st sluice. This value is dominated by our experimental resolution. The small pellet-beam divergence is a consequence of the extremely regular jet breakup in the TPC (Fig. 3).

If used as an internal target at a storage ring, our device minimizes unwanted gas loads to the accelerator and allows for an effective target thickness of a few $10^{15}$ atoms $/ \mathrm{cm}^{2}$. Since the tube connecting the generator with the interaction zone is rather narrow, detectors can be placed close to the interaction point in a nearly $4 \pi$ configuration. Such a geometry is also advantageous for placing collector mirrors in laser-plasma uv sources [9]. In addition, the large separation of the hot and radiating plasma from the jet generator makes such a setup especially favorable.

In this Letter we present first data on the monodisperse breakup of cryogenic jets. We find that the jets are significantly more resistant to breakup than predicted by Rayleigh's theory. We suggest that a reason for this discrepancy is the influence of evaporation effects. This interpretation is supported by the observation of nonaxially symmetric jets. This new jet mode-for which so far not even a rudimentary theoretical explanation has been formulated - sets in when one decreases the ambient pressure very close to the triple-point values. For a complete theoretical delineation of drop formation processes from thin cryogenic jets, further systematic data, such as that on the $X$ dependence of $\gamma / \gamma_{0}$ and the pressure dependence of $L_{\text {jet }}$, are required.

This work has been supported by BMBF (WTZ), COSYFFE, DFG, EU (FP6), INTAS, ISTC, RFFI (07-08-00747a, 08-08-91950-NNIO_a), RMS, and the PANDA Collaboration. The Russian team members are thankful for the hospitality at the FZJ, in particular for the support by H. Ströher. We acknowledge discussions with H. Calen, C. Ekström, and Ö. Nordhage

*m.buescher@fz-juelich.de

[1] F. Savart, Ann. Chim. (Paris) 53, 337 (1833).

[2] Lord Rayleigh, Proc. London Math. Soc. s1-10, 4 (1878).

[3] Lord Rayleigh, Philos. Mag. 34, 145 (1892).
[4] S. Chandrasekhar, Hydrodynamic and Hydromagnetic Stability (Oxford University, New York, 1961), p. 537.

[5] J. Eggers, Rev. Mod. Phys. 69, 865 (1997).

[6] S. P. Lin and R. D. Reitz, Annu. Rev. Fluid Mech. 30, 85 (1998).

[7] A. Kalaaji, B. Lopez, P. Attane, and A. Soucemarianadin, Phys. Fluids 15, 2469 (2003).

[8] O. A. Basaran, AIChE J. 48, 1842 (2002).

[9] B. A. M. Hansson and H. M. Hertz, J. Phys. D 37, 3233 (2004).

[10] S. Ter-Avetisyan, M. Schnürer, P. V. Nickles, M. Kalashnikov, E. Risse, T. Sokollik, W. Sandner, A. Andreev, and V. Tikhonchuk, Phys. Rev. Lett. 96, 145006 (2006).

[11] Ö. Nordhage, Z.-K. Li, C.-J. Friden, G. Norman, and U. Wiedner, Nucl. Instrum. Methods Phys. Res., Sect. A 546, 391 (2005).

[12] E. P. Muntz and M. Dixon, J. Spacecr. Rockets 23, 411 (1986).

[13] N. Ashgriz and F. Mashayek, J. Fluid Mech. 291, 163 (1995); R. Suryo, P. Doshi, and O. A. Basaran, Phys. Fluids 18, 082107 (2006).

[14] V. V. Blazhenkov, A.F. Ginevskii, V.F. Gunbin, A. S. Dmitriev, and S. I. Tscheglov, Fluid Dyn. 30, 544 (1995).

[15] V. V. Blazhenkov, A.F. Ginevskii, V.F. Gunbin, A. S. Dmitriev, and A.I. Motin, J. Eng. Phys. Thermophys. 55, 994 (1988).

[16] V. V. Blazhenkov, A. F. Ginevskii, V. F. Gunbin, and A. S. Dmitriev, Fluid Dyn. 23, 203 (1988); V. V. Blazhenkov, A. F. Ginevskii, V.F. Gunbin, A. S. Dmitriev, and A.I. Shcheglov, Fluid Dyn. 28, 338 (1993).

[17] W. T. Pimbley and H. C. Lee, IBM J. Res. Dev. 21, 21 (1977).

[18] Ö. Nordhage, Ph.D. thesis, Acta Universitatis Upsaliensis, 2006, http://publications.uu.se/abstract.xsql?dbid=7137.

[19] Russian Federation Patent No. 2298890; German Patent Application No. 102007017 212.7-13.

[20] D. U. Hamburg and N.F. Dubrovkin, Hydrogen: Properties, Generation, Storage, Transport, Application (Chemistry Publishing, Moscow, 1989), pp. 109, 142, 174 (in Russian).

[21] Handbook of Physical and Technical Fundamentals of Cryogenics, edited by M.P. Malkov (Energoatomizdat, Moscow, 1963), pp. 90, 123, 176 (in Russian).

[22] M. Saroka, Y. Guo, and N. Ashgriz, AIAA J. 39, 1728 (2001); S. Savtchenko and N. Ashgriz, Phys. Fluids 17, 112102 (2005).

[23] J. M. Chwalek et al., Phys. Fluids 14, L37 (2002). 